\title{
Sertraline and Citalopram Actions on Gut Barrier Function
}

\author{
Johana Eyzaguirre-Velásquez ${ }^{1}$ María Paz González-Toro ${ }^{1}$. Camila González-Arancibia ${ }^{1}$. Jorge Escobar-Luna ${ }^{1}$. \\ Caroll J. Beltrán ${ }^{2} \cdot$ Javier A. Bravo ${ }^{1} \cdot$ Marcela Julio-Pieper ${ }^{1}$ (i)
}

Received: 25 September 2020 / Accepted: 29 October 2020 / Published online: 12 November 2020

(c) The Author(s) 2020

\begin{abstract}
Introduction Disruption of intestinal barrier is a key component to various diseases. Whether barrier dysfunction is the cause or effect in these situations is still unknown, although it is believed that translocation of luminal content may initiate gastrointestinal or systemic inflammatory disorders. Since trauma- or infection-driven epithelial permeability depends on Toll-like receptor (TLR) activity, inhibition of TLR signaling has been proposed as a strategy to protect intestinal barrier integrity after infection or other pathological conditions. Recently, selective serotonin recapture inhibitors including sertraline and citalopram were shown to inhibit TLR-3 activity, but the direct effects of these antidepressant drugs on the gut mucosa barrier remain largely unexplored.

Materials and methods To investigate this, two approaches were used: first, ex vivo studies were performed to evaluate sertraline and citalopram-driven changes in permeability in isolated intestinal tissue. Second, both compounds were tested for their preventive effects in a rat model of disrupted gut barrier, induced by a low protein (LP) diet.

Results Only sertraline was able to increase transepithelial electrical resistance in the rat colon both when used in an ex vivo $(0.8 \mu \mathrm{g} / \mathrm{mL}, 180 \mathrm{~min})$ or in vivo (30 mg/kg p.o., 20 days) fashion. However, citalopram ( $20 \mathrm{mg} / \mathrm{kg}$ p.o., 20 days), but not sertraline, prevented the increase in phospho-IRF3 protein, a marker of TLR-3 activation, in LP-rat ileum. Neither antidepressant affected locomotion, anxiety-like behaviours or stress-induced defecation.

Conclusion Our data provides evidence to support the investigation of sertraline as therapeutic strategy to protect intestinal barrier function under life-threatening situations or chronic conditions associated with gut epithelial disruption.
\end{abstract}

Keywords Gut $\cdot$ Permeability $\cdot$ Barrier $\cdot$ TLR $\cdot$ Sertraline $\cdot$ Citalopram $\cdot$ SSRI

\section{Introduction}

Maintenance of intestinal barrier integrity is essential to a healthy status. Diverse chronic disorders, including inflammatory bowel disease, diabetes, and rheumatologic disorders, are associated with a disruption of intestinal barrier function [1-3]. Moreover, a proportion of patients admitted in intensive care units due to critical illness or injury also display increased gut permeability $[4,5]$ which may elevate

Marcela Julio-Pieper

marcela.julio@pucv.cl

1 Grupo de NeuroGastroBioquímica, Instituto de Química, Facultad de Ciencias, Pontificia Universidad Católica de Valparaíso, Valparaíso, Chile

2 Laboratorio de Inmunogastroenterología, Servicio de Gastroenterología, Hospital Clínico Universidad de Chile, Santiago, Chile the risk of sepsis, multiple organ failure, and probably the risk of death $[6,7]$.

Particularly relevant to mucosal immune function, a family of transmembrane receptors known as Toll-like receptors (TLRs) recognize conserved molecular patterns found in microbial pathogens $[8,9]$. In epithelia, TLRs also participate in injury-induced inflammation and tissue repair mechanisms [10]. In this regard, we have demonstrated that poly(I:C), a TLR-3 agonist, affects gut epithelial permeability in a way that is region-specific: In the rat ileum, poly(I:C) reduced transepithelial electrical resistance, a phenomenon that is interpreted as an increase in tissue permeability [11]. This was accompanied by a reduced expression of claudin 7 (unpublished data by Olavarría-Ramírez 2015), a protein associated both with cell adhesion and tight junction maintenance [12]. On the contrary, in the rat colon, poly(I:C) decreased permeability to macromolecules and prevented epithelial weakening [11]. In the DSS-induced colitis mouse 
model, poly(I:C) exerts a protecting effect in the colon that includes lower disease activity index score and enhanced expression of an important tight junction protein, Zonula occludens 1 (ZO-1) [13].

TLR-3's natural ligand is viral double-stranded RNA, of which poly(I:C) is a synthetic mimic [14]. However, TLR activity can also be modulated by ligands that do not have a microbial origin. In this regard, selective serotonin reuptake inhibitors (SSRIs), which have been known for a while to have anti-inflammatory effects [15-17], have gained attention due to their ability to inhibit TLR activity. The use of a high-throughput screening system allowed identifying the antidepressant sertraline as a TLR-3 inhibitor in vitro [18]. Antidepressant effects on innate immunity have also been demonstrated in an animal model of rheumatoid arthritis: Citalopram exerts potent anti-inflammatory effects that are mediated through TLR-3 inhibition [19]. Together, these data suggest there is great potential for modulating intestinal barrier function by targeting TLR-3 activity with SSRIs such as citalopram or sertraline. Therefore, in this work we aimed to evaluate changes in permeability induced by citalopram or sertraline in isolated intestinal tissue. Also, the protective effect of each agent was tested in a rat model of disrupted gut barrier function, induced by administration of a low protein (LP) diet [20].

\section{Materials and Methods}

\section{Animals}

Male Sprague-Dawley rats were maintained on a $12: 12 \mathrm{~h}$ dark-light cycle with temperature at $20^{\circ} \mathrm{C} \pm 1{ }^{\circ} \mathrm{C}$. Food and water were provided ad libitum for all experiments. Killing was performed by thiopental overdose followed by decapitation. Protocols for animal care and use were approved by the institutional ethics committee of the Pontificia Universidad Católica de Valparaíso, Chile.

\section{Experimental Design}

Three experiments were conducted for this study. For experiment 1 (initial $n=8$ ), ileum and colon from naïve rats were obtained at postnatal day (PND) 60 and exposed to vehicle $(0.9 \% \mathrm{NaCl}$ or saline), citalopram, or sertraline ex vivo. For experiment 2 , rats were fed with a low protein (LP) diet for 5,10 , or 20 days, and all of them were at PND 60 on the last day of treatment, when plasma samples were taken to perform corticosterone assay (initial $n=5,4,4$, and 5 for control diet, 5, 10, and 20 days of LP diet, respectively). For experiment 3 , rats were fed with a low protein (LP) diet for 20 days and were simultaneously given (by means of daily oral gavage) vehicle (saline), citalopram, or sertraline; they were compared to age-matched rats fed a control diet and daily treated with vehicle (20 days, oral gavage). In a first cohort of rats (initial $n=9$ for groups Vehicle and LP, $n=6$ for groups $\mathrm{LP}+\mathrm{S}$ and $\mathrm{LP}+\mathrm{C}$ ) from experiment 3 , intestinal tissue and blood plasma samples were obtained at PND 60 to perform functional/molecular gut tests and corticosterone assay, respectively. A second cohort of rats $(n=4$ for all groups) from experiment 3 was employed to perform an open field test at PND 60. The numbers here stated indicate how many animals were destined to each experiment. Details regarding diet and drug treatments are provided in the following section.

\section{Diet and Drugs}

Control diet was a standard grain-based diet (ProLab RMH3000 from Purina, energy density: $4.1 \mathrm{kcal} / \mathrm{g}$ ). Kilocalories were distributed as follows: protein $25.967 \%$, fat $14.022 \%$, and carbohydrates $60.011 \%$. Experimental, low protein (LP) diet was a custom-made purified diet (Purina, energy density: $4.05 \mathrm{kcal} / \mathrm{g}$ ). Kilocalories were distributed as follows: protein $4.1 \%$, fat $14.9 \%$, and carbohydrates $81.0 \%$. The low protein food was stained blue for easy recognition.

Intestinal tissues obtained for ex vivo experiments ("experiment 1") were taken from rats fed exclusively with control diet. These tissues were exposed to either vehicle (saline), citalopram $(3,30$, or $300 \mu \mathrm{g} / \mathrm{mL})$, or sertraline $(0.8$, 8 or $80 \mu \mathrm{g} / \mathrm{mL}$ ).

In the case of the in vivo time-course experiment ("experiment 2") after weaning at postnatal day (PND) 21, animals from group "0" were fed the control diet until PND 60, being this the day of sample obtaining. Animals from group " 5 " were fed the control diet until PND 54 and then the LP diet from PND 55 until PND 60 (i.e., a total of 5 days). Animals from group "10" were fed the control diet until PND 49 and then the LP diet from PND 50 until PND 60 (i.e., a total of 10 days). Animals from group " 20 " were fed the control diet until PND 39 and then the LP diet from PND 40 until PND 60 (i.e., a total of 20 days).

In the case of the in vivo SSRI experiment ("experiment 3 "), after weaning at postnatal day (PND) 21, all animals were fed the control diet until PND 39. Thereafter, they were separated into the control group, which was kept under the same diet until PND 60, and the low protein (LP) group, which was fed the low protein diet from PND 40 to PND 60 . The LP group was subdivided into vehicle treated (saline daily p.o.), citalopram ( $20 \mathrm{mg} / \mathrm{kg} /$ day p.o.), or sertraline (30 mg/kg/day p.o.). Body weight and health status were monitored daily. 


\section{Tissue Sampling}

Intestinal tissue samples were obtained at PND 60. Fecal contents were rinsed with saline $(0.9 \% \mathrm{NaCl})$. Samples from both ileal and colonic tissue were distributed as follows: (starting from the proximal end of tissue) One $\mathrm{cm}$ was fixed in paraformaldehyde for morphological evaluation, and the next $\mathrm{cm}$ was used to obtain a mucosal scraping. The remaining tissue was divided into two pieces, for Ussing chamber and everted gut sac techniques, respectively.

\section{Transepithelial Electrical Resistance}

The electrical resistance of an epithelial barrier is inversely proportional to its permeability. To measure this parameter, colon and ileum samples previously rinsed with saline were placed in oxygenated $\left(95 \% \mathrm{O} 2,5 \% \mathrm{CO}_{2}\right) \mathrm{Krebs}$ buffer (1.2 $\mathrm{mM} \mathrm{NaH}_{2} \mathrm{PO}_{4}, 117 \mathrm{mM} \mathrm{NaCl}, 4.8 \mathrm{mM} \mathrm{KCl}, 1.2 \mathrm{mM}$ $\mathrm{MgCl}_{2}, 25 \mathrm{mM} \mathrm{NaHCO}_{3}, 2.5 \mathrm{mM} \mathrm{CaCl}_{2}$ and $11 \mathrm{mM}$ glucose). The muscle layers were stripped away, leaving only the mucosa and submucosa. Ileal preparations, which produce a substantial amount of mucus, were pre-incubated in oxygenated Krebs buffer at $37{ }^{\circ} \mathrm{C}$ for 20 min to clear some of this mucus. Preparations were then placed in Ussing chambers (EasyMount from Warner Instruments, Whitehall, PA, USA. Exposed area of $0.3 \mathrm{~cm}^{2}$ ) as described previously [20] with oxygenated Krebs buffer maintained at $37{ }^{\circ} \mathrm{C}$, and voltage-clamped at $0 \mathrm{mV}$ (World Precision Instruments, Sarasota, FL, USA). In the case of colon preparations, these were immediately placed in the Ussing chambers (exposed area of $0.3 \mathrm{~cm}^{2}$ ), as described before [20]. For in vivo experiments, tissues were studied as described; for ex vivo experiments, either vehicle, citalopram $(3,30$, or $300 \mu \mathrm{g} / \mathrm{mL})$, or sertraline $(0.8,8$, or $80 \mu \mathrm{g} / \mathrm{mL})$ was added on the serosal side of ileum and colon preparations. Short-circuit current responses were continuously monitored using LabScribe 2 software (World Precision Instruments). After stabilization, a $2 \mathrm{mV}$ challenge was applied for $5 \mathrm{~s}$ in order to calculate transepithelial electrical resistance (TEER).

\section{Permeability to Macromolecules}

Finding a decrease in TEER is indicative of increased permeability but does not reveal the size of the chemical species that are passing through, which could be ions, small molecules, or even macromolecules. To further investigate this, the permeability to two molecules, with a MW of 4.4 and $40 \mathrm{kD}$, respectively, was assayed. Colon and ileum samples previously rinsed were placed in oxygenated Krebs buffer as described earlier. The procedure was conducted as described in [20]; in brief, tissues were everted using a crochet hook, thereby leaving the mucosa on the outer side. To prepare a sac, one end of a $2-3 \mathrm{~cm}$ everted gut segment was tied and the other end was affixed to a modified $2 \mathrm{~mL}$ cryovial, which allowed for repeated sampling. Each cryovial-intestine unit was then filled with a known volume of Krebs buffer and placed in a $15 \mathrm{~mL}$ centrifuge tube containing oxygenated Krebs buffer. All tubes also contained $30 \mu \mathrm{g} / \mathrm{mL}$ TRITCdextran $4.4 \mathrm{kD}$ (TD4.4) and $30 \mu \mathrm{g} / \mathrm{mL}$ FITC-dextran $40 \mathrm{kD}$ (FD40). Tissues were incubated at $37{ }^{\circ} \mathrm{C}$, and $60 \mu \mathrm{L}$ samples were taken from the internal medium every $60 \mathrm{~min}$ for up to $180 \mathrm{~min}$, which is the maximum time intestinal tissue remains vital in an ex vivo setup [21]. Internal medium samples were diluted 1:10 before performing fluorimetric analyses. Fluorescence (TD4.4: $\lambda$ ex546, $\lambda$ em 575 and FD40: $\lambda$ ex 490, $\lambda$ em520) was measured in a FluoroMax-2 fluorometer (Jobin-Yvon-Spex, Edison, NJ, USA). Standard curves were used to obtain the concentration of each fluorophore (TD4.4 limit of detection: $71 \mathrm{ng} / \mathrm{mL}$ - limit of quantification: $238 \mathrm{ng} / \mathrm{mL}$; FD40 limit of detection: $2 \mathrm{ng} / \mathrm{mL}$-limit of quantification: $7 \mathrm{ng} / \mathrm{mL}$ ). At the end of the assay, the mucosal area was measured. The volume of medium and the exposed mucosal area in each sac were used to express fluorophore transit as pmoles $/ \mathrm{cm}^{2}$ at each sampling time.

\section{Western Blot}

Intestinal mucosa obtained by tissue scraping was homogenized in ice-cold lysis buffer (HEPES $10 \mathrm{mM}, \mathrm{pH}$ 7.9, $\mathrm{KCl} 10 \mathrm{mM}$, EDTA $0.1 \mathrm{mM}$, EGTA $0.1 \mathrm{mM}$, Triton X-100 $0.06 \%$, dithiothreitol $0.5 \mathrm{mM}$ and protease inhibitors PMSF $0.1 \mathrm{mM}, \mathrm{Na} 3 \mathrm{VO} 40.1 \mathrm{mM}, \mathrm{NaF} 0.02 \mathrm{mM}, \mathrm{NaPPi} 0.025 \mathrm{mM}$, leupeptin $2 \mu \mathrm{g} / \mathrm{mL}$, and aprotinin $2 \mu \mathrm{g} / \mathrm{mL}$ ). Protein concentration was determined using Qubit ${ }^{\circledR}$ protein assay (Life Technologies, Carlsbad, CA, USA). $30 \mu \mathrm{g}$ of protein from each mucosal sample was separated by SDS-PAGE (12\%, Sigma, Darmstadt, Germany) and transferred to PVDF membranes (Millipore, Burlington, MA, USA). The membranes were blocked for $1.5 \mathrm{~h}$ at room temperature (Trisbuffered saline containing $0.1 \%$ Tween-20 and 5\% BSA). Mouse anti-total-IRF3 antibody (Santa Cruz, Dallas, TX, USA) or rabbit anti-phospho-IRF3 antibody (Cell Signaling, Danvers, MA. USA) was diluted 1:1000 in blocking solution and applied overnight at $4{ }^{\circ} \mathrm{C}$. After repeated washing, membranes were incubated for $2 \mathrm{~h}$ at room temperature with peroxidase-conjugated anti-mouse or anti-rabbit secondary antibody (1:5000. Jackson ImmunoResearch, West Grove, PA, USA), respectively. Signal was developed using Western-Lightning ${ }^{\circledR}$ Plus-ECL (PerkinElmer, Waltham, MA, USA). Autoradiographic films were scanned and band intensity evaluated by densitometry. For this study, samples were run in singlicate. Membranes were subjected to phospho-IRF3 western blot protocol, then treated for $1 \mathrm{~h}$ at RT with a stripping solution (consisting of $0.02 \%$ Ponceau red, $0.3 \%$ trichloroacetic acid, and $0.3 \%$ sulphosalicylic acid), 
thoroughly washed with PBS-T, and used to detect totalIRF3. Data were analyzed blindly using Multi Gauge software (Fuji Film, Tokyo, Japan) and expressed as the ratio of phospho-IRF3 ( ${ }_{\mathrm{P}} \mathrm{IRF} 3$ ) to total-IRF3 ${ }_{\mathrm{T}} \mathrm{IRF} 3$ ) band intensity.

\section{Immunofluorescence and Confocal Microscopy}

Colon and ileum segments previously rinsed with saline were fixed overnight in $10 \mathrm{mM}$ PBS containing $4 \%$ paraformaldehyde, dehydrated in PBS containing $30 \%$ sucrose, and stored at $-80^{\circ} \mathrm{C}$. The frozen tissue was brought to $-20{ }^{\circ} \mathrm{C}$ to be cut into transverse sections $(4 \mu \mathrm{m}$ thick) and mounted on glass slides. After antigen retrieval (incubation in $10 \mathrm{mM}$ citrate buffer, ph $6,5 \mathrm{~min}$ at $90{ }^{\circ} \mathrm{C}$ and then at 5 min at RT), tissues were washed in PBS containing Triton X-100 $(0.3 \%)$, blocked with a protein block serum free (Dako), and incubated overnight at $4{ }^{\circ} \mathrm{C}$ with the primary antibody (Rabbit anti-ZO-1 from Invitrogen) diluted 1:200 in Dako blocking solution. The secondary antibody (Alexa Fluor 488 donkey anti-rabbit) was diluted 1:1000 in PBS-T and applied for $1 \mathrm{~h}$ at RT. Tissues were then washed and nuclei stained with $1 \mu \mathrm{M}$ DAPI solution (Invitrogen), $2 \mathrm{~min}$ at RT, and coverslips were applied using Prolong Diamond (Molecular Probes/LifeTechnologies).

Stained sections were observed using a Carl Zeiss (LSM 700) confocal microscope, under ZEN control software. Photographs were captured at 40X and analyzed with ImageJ 1.50i. Five photographs from each three non-consecutive sections were evaluated per animal. ZO-1 signal was identified as positive immunofluorescent dots located at the apical side and between enterocytes. ZO-1 signal dots were counted along the epithelium, and results were expressed as number of positive signals $/ \mu \mathrm{m}$ of epithelium.

\section{Open Field (OF)}

This test was performed as described before [22]. Briefly, at postnatal day 60 , rats ( $n=4$ per group) were individually placed in a $60 \times 60 \mathrm{~cm}$ apparatus and behavior was recorded for $5 \mathrm{~min}$ with a camera located $1.5 \mathrm{~m}$ above the test site. After each test, the apparatus was cleaned with 5\% ethanol. Once all videos were acquired, manual quantification was carried out in a blind fashion. The arena was divided into 16 squares, where the inner 4 squares determined the central area of the arena. From the OF test, the following parameters were evaluated to identify stress-related behaviors: fecal pellet output, time spent in the center, and time remained in the peripheral zone.

\section{Corticosterone Enzyme-Linked Immunosorbent Assay (ELISA)}

Blood was collected upon decapitation and was obtained from rats without stress. A commercially available ELISA kit was used to quantify the levels of the stress hormone corticosterone in the plasma according to the manufacturer's instructions (Corticosterone kit, Enzo LifeSciences, NY, USA).

\section{Statistical Analyses}

Values are expressed as mean \pm SEM. Ex vivo TEER data were analyzed by nonparametric 2-way ANOVA, and any overall statistical differences were further examined using Bonferroni test (GraphPad Prism 5 Software Inc. GraphPad Software, San Diego, CA, USA). In vivo TEER data, macromolecule permeability, western blot, and immunofluorescence data were analyzed by nonparametric 1-way ANOVA, and any overall statistical differences were further examined using Dunn's multiple comparison test. (GraphPad Prism 5 Software Inc. GraphPad Software, San Diego, CA, USA). Significance was established at $p<0.05$. Importantly, at the end of the functional assays, only tissues displaying healthy features were included in the analysis. The $\mathrm{n}$ numbers representing data that we were able to retrieve from each experience are shown in each figure legend.

\section{Results}

\section{Ex Vivo Effects of SSRIs on the Permeability of Colon Tissue}

As a first approach, gut preparations from healthy rats were exposed to citalopram $(0.3,3$ or $30 \mu \mathrm{g} / \mathrm{mL})$ or sertraline $(0.8$ or $8 \mu \mathrm{g} / \mathrm{mL}$ ) ex vivo and TEER was registered for up to $3 \mathrm{~h}$. This allowed us to identify acute effects of each drug on healthy gut tissue. For sertraline, the lowest dose induced a significant increase in colon TEER $(F(2,84)=11.57$; $p<0.0001)$. Also, a Bonferroni posttest revealed that the highest dose had a significant effect at the $3 \mathrm{~h}$ time point $(p<0.05)$; in the case of citalopram, colon tissues treated with the highest dose also had a higher TEER than vehicletreated tissues, but in a nonsignificant fashion (see Fig. 1a, b). In contrast to colonic tissue, ileal tissue preparations rapidly deteriorated under ex vivo treatment with sertraline and citalopram, and therefore, those data are not reported. Similar deterioration was observed in colon preparations treated with $80 \mu \mathrm{g} / \mathrm{mL}$ sertraline (not shown). 


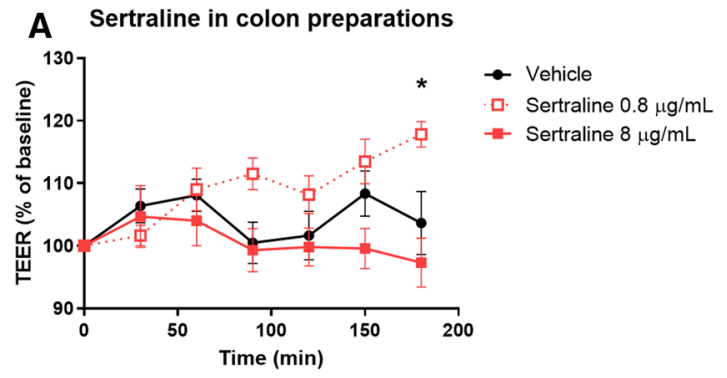

Fig. 1 TEER of gut preparations treated with SSRIs ex vivo. a Sertraline $(0,0.8$, or $8 \mu \mathrm{g} / \mathrm{mL})$ treated colon tissue ( $n=5$ for all groups). b Citalopram $(0,0.3,3$, or $30 \mu \mathrm{g} / \mathrm{mL})$ treated colon tissue $(n=5,4,4$,

\section{Stress-Associated Phenotype in Rats Subjected to LP Diet With and Without SSRIs}

It has been previously reported by others and by us that low protein (LP) diet results in disruption of ileal and colonic barrier integrity $[20,23,24]$. We wanted to evaluate the influence of two SSRIs on gut epithelial barrier, without having confounding systemic effects such as stress-induced hyperpermeability. Therefore, we verified whether the phenotype associated with 1) a 20-day LP diet and 2) a 20-day LP diet plus daily sertraline $(30 \mathrm{mg} / \mathrm{kg} /$ day $)$ or citalopram $(20 \mathrm{mg} / \mathrm{kg} /$ day) involved behavioral or endocrine alterations associated with stress. Figure 2a shows that corticosterone levels were not significantly increased along LP treatment in naïve rats, in comparison with rats fed a regular chow. Also, neither citalopram nor sertraline was able to modify corticosterone levels in the plasma of LP-treated rats (Fig. 2b). As expected, basal corticosterone levels in vehicle rats subjected to oral gavage (Fig. 2b) were higher than in control diet naïve rats (Fig. 2a). In the open field, LP rats had a locomotor activity and fecal pellet output that was not different from vehicle rats (Fig. 2c, d). Likewise, these parameters were not affected by sertraline or citalopram. Regarding the time spent in the center and periphery of the open field, no effect was observed under LP diet or LP diet plus an SSRI (see Fig. 2e, f). Reference values for stress-induced fecal pellet output in the open field as well as differences in time spent in the center and periphery of the open field as result of anxiogenic experimental conditions can be found in $[22,25]$.

\section{In Vivo Effects of SSRIs on Gut TEER and Permeability to Macromolecules}

In both ileum and colon from rats subjected to LP diet, sertraline prevented the reduction in baseline mucosal TEER (see Fig. 3a, b); however, the effect was statistically significant only in the colon. Citalopram did not prevent the reduction in baseline mucosal TEER in either ileum or colon from rats subjected to LP diet, although there is a similar

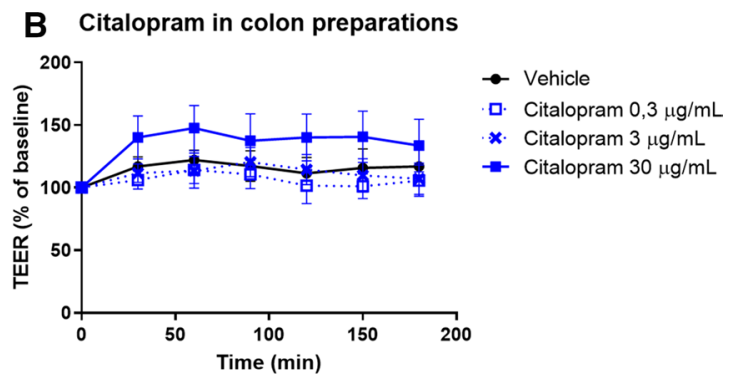

and 5, respectively). Data are expressed as mean \pm SEM. ${ }^{*} p<0.05$ in Bonferroni test

pattern to what was observed with sertraline. After $3 \mathrm{~h}$ of culture, permeability of ileum or colon to 4.4 and $40 \mathrm{kDa}$ dextrans was not different between LP diet-treated and vehicle rats. Neither sertraline nor citalopram induced a significant change in the gut permeability to these macromolecules (see Fig. 4a-d).

\section{In Vivo Effects of SSRIs on Zonula Occludens-1 (ZO-1) in the Gut Mucosa}

The density of pixels corresponding to the immunoreactive signal of ZO-1 (a protein found in tight junctions) in ileum and colon mucosa was not modified by either LP diet or LP diet plus sertraline or citalopram (not shown). However, when counting the number of discrete spots of positive ZO-1 immunoreactive signal along the mucosal epithelium (see Fig. 5a), there was an overall effect of LP diet, but only in the ileum (Fig. 5b, c). Moreover, the ileum of the LP-treated plus citalopram group was the only to display a significant decrease in the amount of stained regions, in comparison with the vehicle group (Fig. 5b).

\section{In Vivo Effects of SSRIs on the Abundance of Phospho-IRF3 in the Gut Mucosa}

In order to assess whether modulation of intestinal barrier function with citalopram or sertraline is associated with inhibition of TLR-3 activity, we looked at IRF3, a signaling molecule that is modified by phosphorylation upon TLR-3 activation. LP diet on its own induced a significant increase in the relative amount of phospho-IRF3 to totalIRF3 ( $\mathrm{P} I R F 3 / \mathrm{T} I R F 3)$ detected in mucosal scrapings from both ileum and colon (Fig. 6a). In the ileum, citalopram (but not sertraline) treatment prevented this increase in LP diet-treated rats. However, in the colon, neither sertraline nor citalopram treatment was able to modify the levels of ${ }_{\mathrm{P}} \mathrm{IRF} 3$ in the gut mucosa (Fig. 6b). 

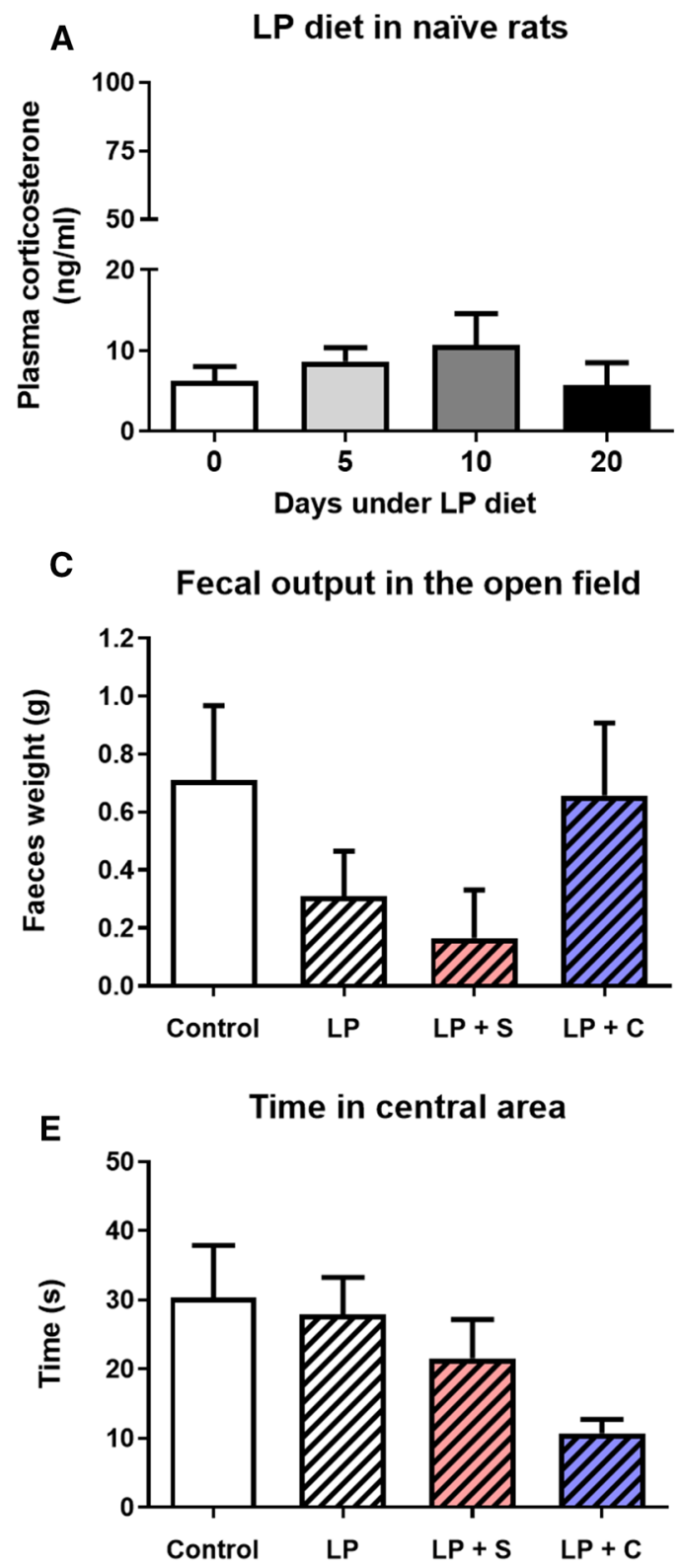

Fig. 2 Evaluation of physiological and behavioral markers of stress in rats fed a LP diet. a Time-course response of naïve rats under LP diet $(n=5,3,4$, and 5 for control diet, 5, 10, and 20 days of LP diet, respectively). b Corticosterone levels in rats that had a 20-day treatment with either: control diet plus vehicle (Vehicle), LP diet plus

\section{Discussion}

Antidepressants have been used for some time to improve gastrointestinal (GI) function in patients [26] being the main focus restoration of normal GI motility. Little is known about the direct effects of antidepressant drugs on the gut mucosa or the potential actions of antidepressant treatment on the intestinal barrier. The present work reports, for the first time, the in vivo effects of two SSRIs on diverse aspects of the rat gut barrier function.

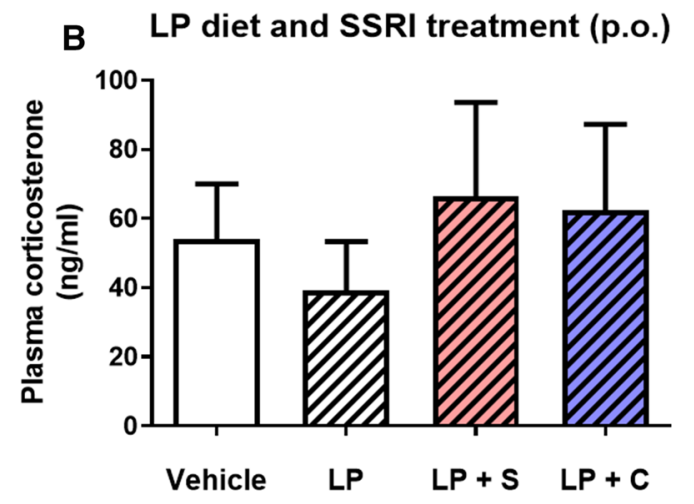

D

Distance traveled
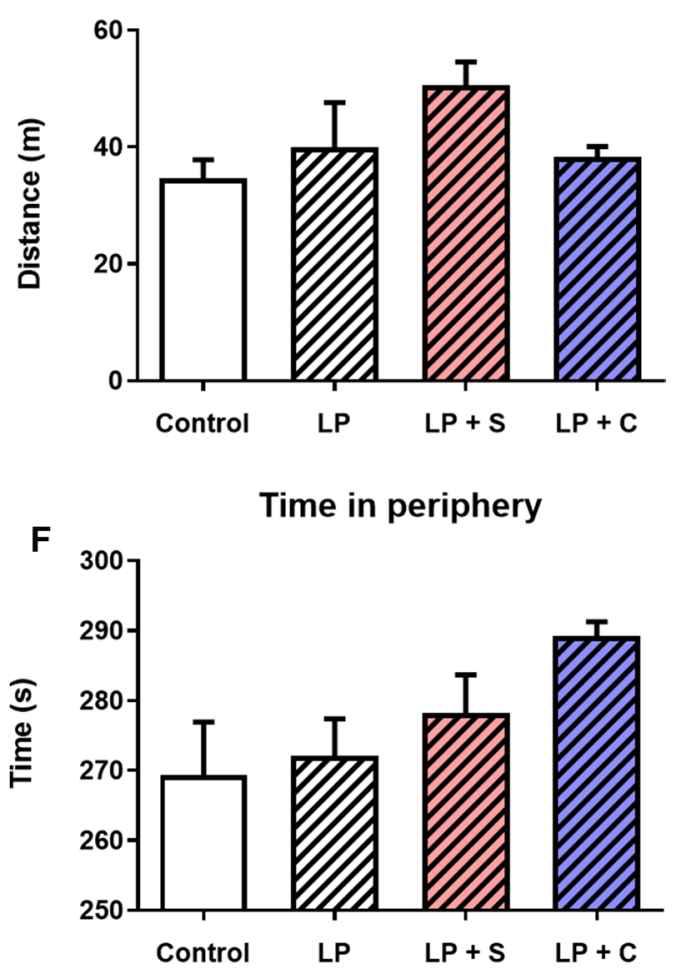

vehicle (LP), LP diet plus sertraline at $30 \mathrm{mg} / \mathrm{kg} / \mathrm{day}(\mathrm{LP}+\mathrm{S})$, or LP diet plus citalopram at $20 \mathrm{mg} / \mathrm{kg} /$ day $(\mathrm{LP}+\mathrm{C})(n=7,7,5$, and 4 , respectively). c-f Behavioral outcomes of LP rats under SSRI treatment when tested in the open field ( $n=4$ for all groups). Data are expressed as mean $\pm \mathrm{SEM}$

For this study, a suitable model of dysfunctional gut barrier was required to show no signs of physiological stress. There were two reasons for this: First, stress-induced hyperpermeability could promote confounding systemic effects. Second, we wanted to distinguish the direct actions of antidepressants on the epithelial barrier from those potentially induced indirectly, by relieving stress. We had previously used a model based on protein malnutrition which displayed decreased transepithelial electrical resistance both at the ileum and colon [20], although we were unaware of potential 


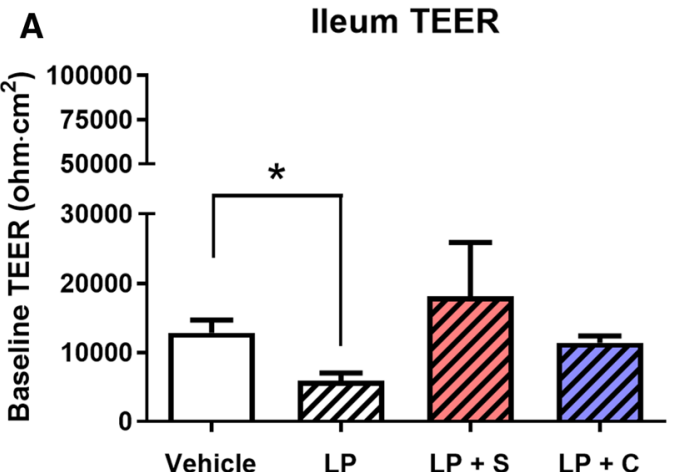

Fig. 3 Transepithelial electrical resistance (TEER) of gut preparations obtained from animals that had a 20-day treatment with either: control diet plus vehicle (Vehicle), LP diet plus vehicle (LP), LP diet plus sertraline at $30 \mathrm{mg} / \mathrm{kg} / \mathrm{day}(\mathrm{LP}+\mathrm{S})$, or LP diet plus citalopram at $20 \mathrm{mg} / \mathrm{kg} / \mathrm{day}(\mathrm{LP}+\mathrm{C})$. a Ileum TEER $(n=5,4,3$, and 5 , respec-

\section{B}

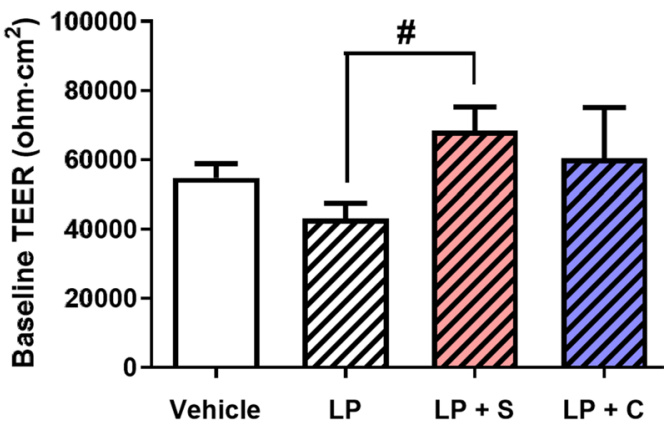

tively). b Colon TEER ( $n=5,5,5$, and 4 , respectively). In both (a) and (b), a posttest was performed to compare LP with vehicle, and $\mathrm{LP}+\mathrm{S}$ or $\mathrm{LP}+\mathrm{C}$ with $\mathrm{LP}$ group. Data are expressed as mean $\pm \mathrm{SEM}$. $* p<0.05 \mathrm{LP}$ versus Vehicle and ${ }^{\#} p<0.05 \mathrm{LP}+\mathrm{S}$ versus LP in Dunn's multiple comparison test
Fig. 4 Permeability to macromolecules in gut preparations obtained from animals that had a 20-day treatment with either: control diet plus vehicle (Vehicle), LP diet plus vehicle (LP), $\mathrm{LP}$ diet plus sertraline at $30 \mathrm{mg} /$ $\mathrm{kg} /$ day $(\mathrm{LP}+\mathrm{S})$, or LP diet plus citalopram at $20 \mathrm{mg} / \mathrm{kg} /$ day $(\mathrm{LP}+\mathrm{C})$. a Ileum permeability to $4.4 \mathrm{kD}$ dextran $(n=9,6,6$, and 4 , respectively). b Ileum permeability to $40 \mathrm{kD}$ dextran ( $n=6,7,5$, and 4 , respectively). c Colon permeability to $4.4 \mathrm{kD}$ dextran $(n=9,9,5$, and 4 , respectively). d Colon permeability to $40 \mathrm{kD}$ dextran $(n=7$, 7,5 , and 4 , respectively). Data are expressed as mean \pm SEM
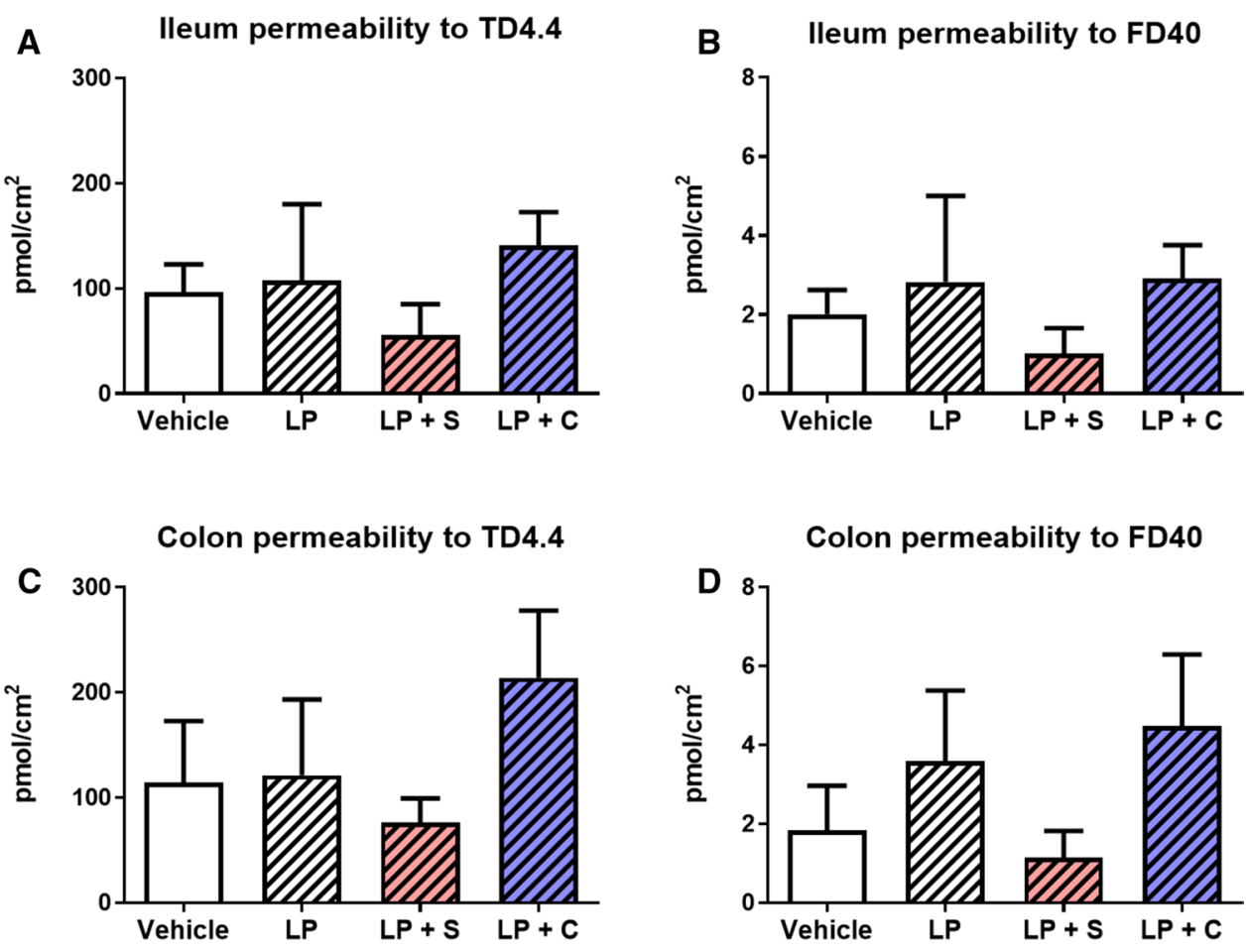

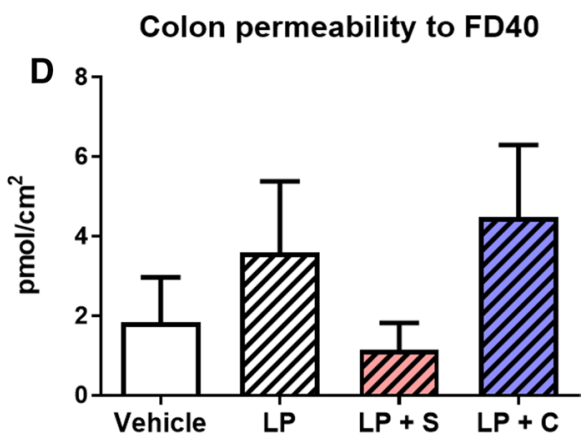

effects on the rat's stress response. The low protein (LP) diet seemed to induce an early, nonsignificant increase in plasma corticosterone; however, by 20 days of diet, corticosterone levels were not different from those found in animals fed regular chow. Moreover, LP-fed rats did not show anxietylike behaviors in the open field, in comparison with regular chow-fed controls, which would appear as increased fecal output, increased time in the periphery, and decreased time in the central area $[22,25]$. Rats subjected to daily oral gavage had higher plasma corticosterone levels than naïve rats (compare control groups from Fig. 2a, b), although rats in the $\mathrm{LP}, \mathrm{LP}+\mathrm{S}$, and $\mathrm{LP}+\mathrm{C}$ groups were not under the effect of higher corticosterone levels than their own control group (vehicle-treated rats). Furthermore, SSRI treatment had no statistically significant effect on behavior and motor activity. These data allowed us to define this model as suitable for the present investigation. In order to be precise, we must draw attention to the fact that in our LP diet, proteins were replaced by carbohydrates. In addition, unlike the control diet, which is grain based, the LP diet here used was custommade from purified nutrients which contain substantially less fiber. Therefore, it is likely that the alterations reported 
A

ZO-1 in Ileum

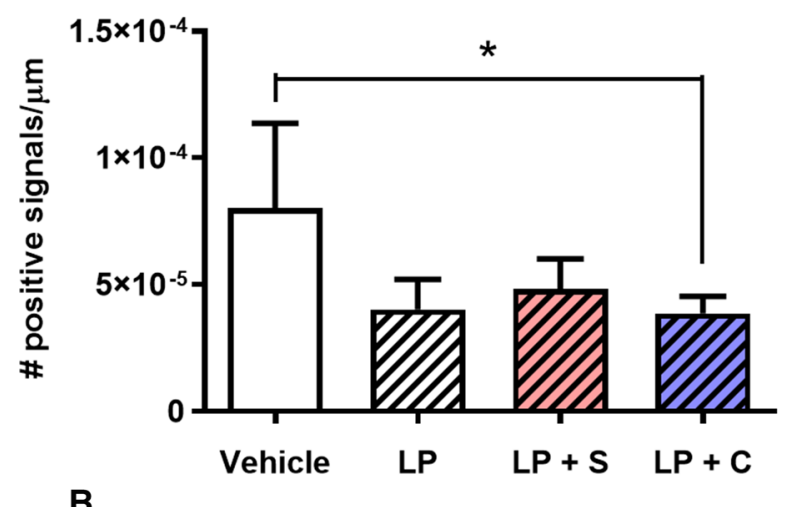

ZO-1 in Colon
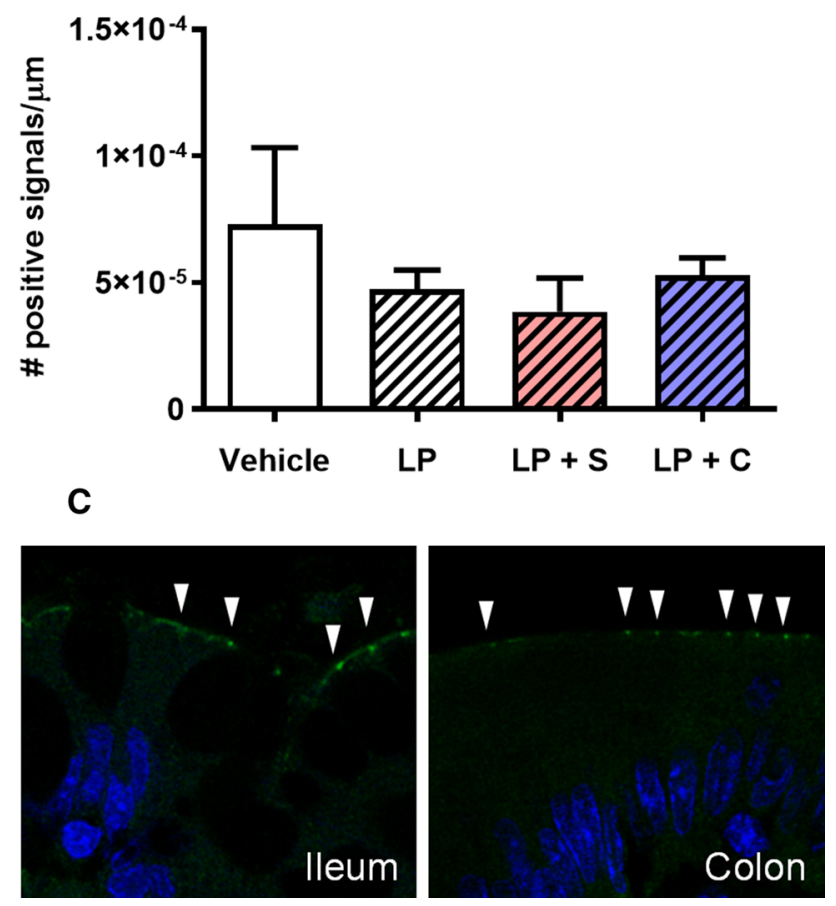

Fig. 5 ZO-1 immunoreactive signal in gut sections obtained from rats fed a LP diet. a Representative image of ZO-1 staining observed in the gut mucosa. The arrowheads mark positive $\mathrm{ZO}-1$ signals (green). Cell nuclei are stained blue. b ZO-1 signals in ileum from rats that had a 20-day treatment with either: control diet plus vehicle (Vehicle), LP diet plus vehicle (LP), LP diet plus sertraline at $30 \mathrm{mg} / \mathrm{kg} /$ day $(\mathrm{LP}+\mathrm{S})$, or LP diet plus citalopram at $20 \mathrm{mg} / \mathrm{kg} / \mathrm{day}(\mathrm{LP}+\mathrm{C})$ ( $n=4$ for all groups). c ZO-1 signals in colon from the same experimental groups described in (b) $(n=3,3,4$, and 4, respectively). Data are expressed as mean \pm SEM. ${ }^{*} p<0.05$ vs vehicle in Dunn's multiple comparisons test

here are the result of a combination of effects driven by low protein ( $4.1 \%$ of energy vs. $26 \%$ in control diet) high carbohydrate ( $81 \%$ of energy vs. $60 \%$ in control diet), and low fiber content (3.9\% of mass vs. $24.5 \%$ in control diet), including the corresponding repercussions in gut microbiota.
The present investigation was addressed to citalopram and sertraline, two of the three SSRI drugs with reported anti-inflammatory effects associated with TLR-3 inhibition $[18,19]$. Fluoxetine was the third compound with such reported effects; however, we left it out of this study because it showed no effect on our exploratory ex vivo gut permeability assays (data not shown). For our ex vivo experiments, we tested a range of concentrations including those reported to have anti-inflammatory effects $[18,19]$ and we found that sertraline induced a small, significant reduction in colon permeability, and citalopram generated a larger, although not statistically significant effect, which led us to proceed with the in vivo experiments. Also, citalopram was used instead of escitalopram; while the S-enantiomer has been described to have a higher potency than the racemic mixture citalopram in reducing anxiety-like behaviors in rodents [27] as well as a greater efficacy in outpatient treatment of major depressive disorder [28], no such descriptions are available regarding TLR-3 binding and the subsequent anti-inflammatory effects. Therefore, we decided to use the racemic mixture that was proven by [19] to have TLR-3 inhibiting properties. Oral instead of intraperitoneal dosage of drugs was selected to ensure a good availability to the GI tract and hence to the intestinal mucosa, considering that significant fecal elimination is suggested for both oral citalopram and sertraline [29]. The doses here used were based on two reports showing anti-inflammatory effects of SSRIs in rodent models of rheumatoid arthritis [19, 30]. Most SSRIs, including sertraline and citalopram, have a half-life of 24-36 $\mathrm{h}$ in humans. In rats, orally administered sertraline has been reported to have a half-life of $4 \mathrm{~h}$ [31], remarkably shorter than in humans. On the other hand, the half-life of citalopram in rats is $1-1.6 \mathrm{~h}$ when the drug is administered intraperitoneally; lower bioavailability is expected for the oral route, due to increased biotransformation in this animal model [32]. The enhanced metabolism of these two compounds in rats, in comparison with humans, has been described by several authors [32-35]. Moreover, Velez de Mendizabal and collaborators declare having unpublished data showing that citalopram metabolic rate by rat microsomes is 18 -fold higher than in human microsomes [32]. Considering this, the oral doses of sertraline and citalopram used in the present work should be considered sub-effective in terms of behavior. Under this criterion, we decided to exclude two control groups from our design: Control diet $+\mathrm{C}$ and control diet $+\mathrm{S}$, although we acknowledge this may be considered a caveat of the present study.

It has been previously shown that gut barrier defects are accompanied by decreased expression of tight junction proteins such as ZO-1 [36]. Alternatively, reduced apical localization of ZO-1 has also been described [37]. We observed a reduction in the number of $\mathrm{ZO}-1$ immunoreactive sites in the gut mucosa of rats that were fed LP diet; however, 

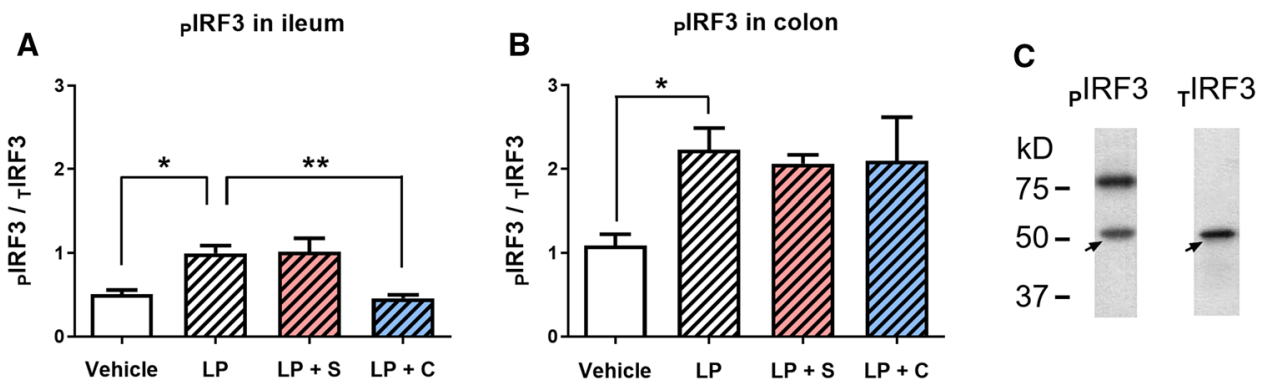

Fig. 6 Phospho-IRF3 abundance (relative to that of total-IRF3, expressed as ${ }_{\mathrm{P}} \mathrm{IRF} 3 / \mathrm{T}_{\mathrm{T}} \mathrm{RF} 3$ ) in gut mucosa from animals that had a 20-day treatment with either: control diet plus vehicle (Vehicle), LP diet plus vehicle (LP), LP diet plus sertraline at $30 \mathrm{mg} / \mathrm{kg}$ / day $(\mathrm{LP}+\mathrm{S})$, or $\mathrm{LP}$ diet plus citalopram at $20 \mathrm{mg} / \mathrm{kg} / \mathrm{day}(\mathrm{LP}+\mathrm{C})$. a Ileum $(n=7,9,6$ and 4 , respectively). b Colon $(n=6,9,6$, and 4 , respectively). In both (a) and (b), a posttest was performed to compare LP with vehicle, and $\mathrm{LP}+\mathrm{S}$ or $\mathrm{LP}+\mathrm{C}$ with $\mathrm{LP}$ group. Data are expressed as mean \pm SEM. $* p<0.05$ versus vehicle. $* * p<0.01$ versus LP in Dunn's multiple comparisons test. $\mathbf{c}$ Representative image of a western blot for phospho- and total-IRF3 protein in a colon mucosa sample sertraline-induced barrier protection such as that observed in the colon must follow a different mechanism, as the effect on colon ZO-1 signal was not prevented in $\mathrm{LP}+\mathrm{S}$ (or in $\mathrm{LP}+\mathrm{C}$ ) animals. Therefore, future studies should include a more comprehensive evaluation of other proteins found in intestinal tight junctions, such as occludin and claudins, to name a few [38].

According to the literature, SSRIs are able to attenuate proinflammatory responses in vitro [39] and in vivo [40]. Modulation of gut barrier function has been suggested as a mechanism behind an individual's inflammatory status; therefore, we expected to observe a reinforcement of the epithelial barrier in gut tissues treated with either sertraline or citalopram. However, the effects of the SSRI compounds here tested were markedly different from each other. Whereas sertraline improved TEER in the colon both ex vivo and in vivo, citalopram had no significant effect. On the other hand, only citalopram was able to prevent, the LP dietinduced increase in phospho-IRF3, a signaling molecule associated with TLR3-induced inflammation [41]. Keeping in mind that the present study did not evaluate inflammation markers, these data not only imply that the search for a mechanism behind sertraline-induced barrier protection must continue, but also are in line with previous evidence suggesting that citalopram anti-inflammatory actions are mediated through TLR-3 inhibition [19]. While the drugs here studied share the same proposed mechanism when used to treat disorders of the central nervous system, our data suggest this is not the case when targeting the gut barrier function, although the implicated mechanism is not within the scope of this study.

Data on TLR-3 binding to DAMPs (damage-associated molecular patterns generated by cells) are solid but still very limited: Upon radiation-induced cell damage, non-coding eukaryotic RNA (such as U1 RNA) is released. The size of intact U1 RNA is about $160 \mathrm{nt}$, but after irradiation, fragments smaller than $100 \mathrm{nt}$ are produced; these molecules are able to bind TLR-3 and elicit TLR-3-dependent signaling [42]. However, no data are available regarding the ability of TLR-3 to bind smaller dsRNA molecules, such as 20-30 nt miRNAs or siRNAs generated by Dicer activity [43]. This is unlikely according to crystallography data [44] and binding affinity studies [45] which show that, in order to form a stable complex with TLR-3, a dsRNA oligonucleotide must be at least 40-50 nt in length. Therefore, although certain dsRNA from the host can be recognized by TLRs, the ability of SSRIs to interfere with signaling elicited by TLR-3 binding to self-ligands (that is, in addition to viral or viral-like particles) remains to be investigated.

From the two types of functional assays that were performed, only those evaluating TEER showed significant effects of either LP diet or SSRI compounds. No changes were observed in the macromolecule permeability assays, on the other hand (Fig. 4). This may be interpreted as indicative of alterations in the gut barrier that affect the passage of molecules smaller than $4.4 \mathrm{kD}$, which was the $\mathrm{MW}$ of the smallest fluorescent tracer here used.

Ileal and colonic tissues displayed similar effects under LP diet, but not always did they show the same response to a particular SSRI. For example, in our ex vivo experiments both sertraline and citalopram seemed to induce rapid deterioration of ileal preparations, an effect that was not observed in colonic samples, with the exception of the highest dose of sertraline $(80 \mu \mathrm{g} / \mathrm{mL})$. Citalopram was able to prevent the increase in phospho-IRF3 induced by LP diet only in the ileum, but not in the colon, where relative phospho-IRF3 protein levels were similar to those induced by LP diet. Divergent results between these gut regions are not entirely unexpected, considering the fact that their microbiota composition, which ultimately shapes mucosal immunity, is markedly different [46]. 
SSRIs are under continuous investigation not only for alternative uses but also to discover novel targets and mechanisms explaining their currently known effects. Strong correlations have been found between the abundance of depression-related bacteria and anxiety- and depressionlike behaviors in mice subjected to chronic unpredicted mild stress [47]. In that study, fluoxetine $(12 \mathrm{mg} / \mathrm{kg} /$ day p.o.) restored gut dysbiosis induced by stress in mice, being able to correct the variation of depression-related bacteria [47]. In a similar focus, another study reported that gut bacteria transplanted from chronically stressed mice to naïve ones were able to induce a depressive phenotype and minimize fluoxetine $(18 \mathrm{mg} / \mathrm{kg} /$ day) efficacy in the recipient via alterations in serotonin and tryptophan metabolism [48]. To date, no such data are available for sertraline or citalopram; however, if this was also the case for these two compounds, at least two types of mechanisms would be worth exploring regarding intestinal barrier protection: short term, potentially dysbiosis-independent effects, accounting for the ex vivo observations, and long term, potentially dysbiosis-related effects, accounting for the in vivo results here reported.

To conclude, the present work shows that citalopram and sertraline are able to affect gut mucosal function by either altering epithelial barrier properties, modifying tight junction protein localization, or changing the level of postraductional modifications in signaling proteins. Moreover, this investigation provides evidence supporting further investigation of these SSRIs as therapeutic strategy to protect intestinal barrier function under life-threatening situations or pathological conditions associated with gut barrier disruption.

Author's contribution MJ and JEV established the experimental design. JEV and JEL conducted the in vivo experiments, including animal treatment and behavioral tests. JEV, MG, and CG performed the ex vivo experiments, including permeability assays and western blot analyses. JEV and CB carried out the immunofluorescent analyses. JEV, MJ, and JB performed the statistical analyses. MJ and JB drafted the manuscript. All authors revised and approved the final version of the manuscript.

Funding This study was funded by Fondecyt 1130213 and Fondecyt 1181019 .

\section{Compliance with Ethical Standards}

Conflict of interest The authors declare that they have no conflict of interest.

Ethical approval All applicable international, national, and/or institutional guidelines for the care and use of animals were followed. All procedures performed in studies involving animals were in accordance with the ethical standards of the institution at which the studies were conducted.

Human and animal rights statement This article does not contain any studies with human participants performed by any of the authors.
Open Access This article is licensed under a Creative Commons Attribution-NonCommercial 4.0 International License, which permits any non-commercial use, sharing, adaptation, distribution and reproduction in any medium or format, as long as you give appropriate credit to the original author(s) and the source, provide a link to the Creative Commons licence, and indicate if changes were made. The images or other third party material in this article are included in the article's Creative Commons licence, unless indicated otherwise in a credit line to the material. If material is not included in the article's Creative Commons licence and your intended use is not permitted by statutory regulation or exceeds the permitted use, you will need to obtain permission directly from the copyright holder. To view a copy of this licence, visit $\mathrm{http} / / /$ creativecommons.org/licenses/by-nc/4.0/.

\section{References}

1. Fasano A, Shea-Donohue T. Mechanisms of disease: the role of intestinal barrier function in the pathogenesis of gastrointestinal autoimmune diseases. Nat Clin Pract Gastroenterol Hepatol. 2005;2:416-422.

2. Bosi E, et al. Increased intestinal permeability precedes clinical onset of type 1 diabetes. Diabetologia. 2006;49:2824-2827.

3. Ciccia F, et al. Over-expression of paneth cell-derived anti-microbial peptides in the gut of patients with ankylosing spondylitis and subclinical intestinal inflammation. Rheumatology (Oxford). 2010;49:2076-2083.

4. Aranow JS, Fink MP. Determinants of intestinal barrier failure in critical illness. Br J Anaesth. 1996;77:71-81.

5. Derikx JP, et al. New insight in loss of gut barrier during major non-abdominal surgery. PLoS One. 2008;3:1-7.

6. Doig CJ, et al. Increased intestinal permeability is associated with the development of multiple organ dysfunction syndrome in critically ill ICU patients. Am J Respir Crit Care Med. 1998; 158:444-451.

7. Fink MP. Intestinal epithelial hyperpermeability: update on the pathogenesis of gut mucosal barrier dysfunction in critical illness. Curr Opin Crit Care. 2003;9:143-151.

8. Liew FY, et al. Negative regulation of toll-like receptor-mediated immune responses. Nat Rev Immunol. 2005;5:446-458.

9. Sirard JC, Bayardo M, Didierlaurent A. Pathogen-specific TLR signaling in mucosa: mutual contribution of microbial TLR agonists and virulence factors. Eur J Immunol. 2006;36:260-263.

10. Gribar SC, et al. No longer an innocent bystander: epithelial toll-like receptor signaling in the development of mucosal inflammation. Mol Med. 2008;14:645-659.

11. Moyano-Porcile V, et al. Short-term effects of Poly(I:C) on gut permeability. Pharmacol Res. 2015;101:130-136.

12. Fujita $\mathrm{H}$, et al. Differential expression and subcellular localization of claudin- $7,-8,-12,-13$, and -15 along the mouse intestine. J Histochem Cytochem. 2006;54:933-944.

13. Zhao HW, et al. Effect of toll-like receptor 3 agonist poly I:C on intestinal mucosa and epithelial barrier function in mouse models of acute colitis. World J Gastroenterol. 2017;23:999-1009.

14. Fortier ME, et al. The viral mimic, polyinosinic:polycytidylic acid, induces fever in rats via an interleukin-1-dependent mechanism. Am J Physiol Regul Integr Comp Physiol. 2004;287:R759-R766.

15. Basterzi AD, et al. IL-6 levels decrease with SSRI treatment in patients with major depression. Hum Psychopharmacol. 2005;20:473-476.

16. Sutcigil L, et al. Pro- and anti-inflammatory cytokine balance in major depression: effect of sertraline therapy. Clin Dev Immunol. 2007;2007:1-6. 
17. Bhagwagar Z, Hafizi S, Cowen PJ. Acute citalopram administration produces correlated increases in plasma and salivary cortisol. Psychopharmacology (Berl). 2002;163:118-120.

18. Zhu J, et al. High-throughput screening for TLR3-IFN regulatory factor 3 signaling pathway modulators identifies several antipsychotic drugs as TLR inhibitors. J Immunol. 2010;184:5768-5776.

19. Sacre $\mathrm{S}$, et al. Fluoxetine and citalopram exhibit potent antiinflammatory activity in human and murine models of rheumatoid arthritis and inhibit toll-like receptors. Arthritis Rheum. 2010;62:683-693.

20. Eyzaguirre-Velasquez J, et al. Protein malnutrition during juvenile age increases ileal and colonic permeability in the rat. J Pediatr Gastroenterol Nutr. 2017;64:707-712.

21. Clarke LL. A guide to Ussing chamber studies of mouse intestine. Am J Physiol Gastrointest Liver Physiol. 2009;296:G1151-G1166.

22. Barrera-Bugueno C, et al. Anxiogenic effects of a Lactobacillus, inulin and the synbiotic on healthy juvenile rats. Neuroscience. 2017;359:18-29.

23. Worthington BS, Boatman ES. The influence of protein malnutrition on ileal permeability to macromolecules in the rat. Am J Dig Dis. 1974;19:43-55.

24. Natali MRM, de Miranda Neto MH, Orsi AM. Effects of hypoproteic diet supply on adult Wistar rats (Rattus norvegicus). Acta Scientiarum. 2000;22:567-571.

25. O'Malley D, et al. Distinct alterations in colonic morphology and physiology in two rat models of enhanced stress-induced anxiety and depression-like behaviour. Stress. 2010;13:114-122.

26. Ford AC, et al. Efficacy of antidepressants and psychological therapies in irritable bowel syndrome: systematic review and meta-analysis. Gut. 2009;58:367-378.

27. Fish EW, et al. Anxiolytic-like effects of escitalopram, citalopram, and R-citalopram in maternally separated mouse pups. J Pharmacol Exp Ther. 2004;308:474-480.

28. Moore N, Verdoux H, Fantino B. Prospective, multicentre, randomized, double-blind study of the efficacy of escitalopram versus citalopram in outpatient treatment of major depressive disorder. Int Clin Psychopharmacol. 2005;20:131-137.

29. Hiemke C, Hartter S. Pharmacokinetics of selective serotonin reuptake inhibitors. Pharmacol Ther. 2000;85:11-28.

30. Baharav E, et al. Immunomodulatory effect of sertraline in a rat model of rheumatoid arthritis. Neuroimmunomodulation. 2012;19:309-318.

31. Melis V, Usach I, Peris JE. Determination of sertraline in rat plasma by HPLC and fluorescence detection and its application to in vivo pharmacokinetic studies. J Sep Sci. 2012;35:3302-3307.

32. Velez de Mendizabal N, et al. A population PK model for citalopram and its major metabolite, $N$-desmethyl citalopram, in rats. $J$ Pharmacokinet Pharmacodyn. 2015;42:721-733.
33. Tremaine LM, Welch WM, Ronfeld RA. Metabolism and disposition of the 5-hydroxytryptamine uptake blocker sertraline in the rat and dog. Drug Metab Dispos. 1989;17:542-550.

34. Catterson ML, Preskorn SH. Pharmacokinetics of selective serotonin reuptake inhibitors: clinical relevance. Pharmacol Toxicol. 1996;78:203-208.

35. Fredricson Overo K. Kinetics of citalopram in test animals; drug exposure in safety studies. Prog Neuropsychopharmacol Biol Psychiatry. 1982;6:297-309.

36. Musch MW, Walsh-Reitz MM, Chang EB. Roles of ZO-1, occludin, and actin in oxidant-induced barrier disruption. Am J Physiol Gastrointest Liver Physiol. 2006;290:G222-G231.

37. Hamada K, et al. Zonula Occludens- 1 alterations and enhanced intestinal permeability in methotrexate-treated rats. Cancer Chemother Pharmacol. 2010;66:1031-1038.

38. Suzuki T. Regulation of intestinal epithelial permeability by tight junctions. Cell Mol Life Sci. 2013;70:631-659.

39. Tynan RJ, et al. A comparative examination of the anti-inflammatory effects of SSRI and SNRI antidepressants on LPS stimulated microglia. Brain Behav Immun. 2012;26:469-479.

40. Wang L, et al. Effects of SSRIs on peripheral inflammatory markers in patients with major depressive disorder: a systematic review and meta-analysis. Brain Behav Immun. 2019;79:24-38.

41. Chattopadhyay S, et al. Inhibition of viral pathogenesis and promotion of the septic shock response to bacterial infection by IRF-3 are regulated by the acetylation and phosphorylation of its coactivators. MBio. 2013;4:1-8.

42. Bernard JJ, et al. Ultraviolet radiation damages self noncoding RNA and is detected by TLR3. Nat Med. 2012;18:1286-1290.

43. Svobodova E, Kubikova J, Svoboda P. Production of small RNAs by mammalian Dicer. Pflugers Arch. 2016;468:1089-1102.

44. Liu L, et al. Structural basis of toll-like receptor 3 signaling with double-stranded RNA. Science. 2008;320:379-381.

45. Leonard JN, et al. The TLR3 signaling complex forms by cooperative receptor dimerization. Proc Natl Acad Sci USA. 2008; 105:258-263.

46. Allaire JM, et al. The intestinal epithelium: central coordinator of mucosal immunity. Trends Immunol. 2018;39:677-696.

47. Sun L, et al. Fluoxetine ameliorates dysbiosis in a depression model induced by chronic unpredicted mild stress in mice. Int $J$ Med Sci. 2019;16:1260-1270.

48. Siopi E, et al. Changes in gut microbiota by chronic stress impair the efficacy of fluoxetine. Cell Rep. 2020;30:3682-3690.

Publisher's Note Springer Nature remains neutral with regard to jurisdictional claims in published maps and institutional affiliations. 